\title{
ALMOST HYPERSURFACE STRUCTURES
}

\author{
ROBERT H. BOWMAN \& DONALD H. SINGLEY
}

\section{Introduction}

This paper is a continuation of the paper, Almost submanifold structures, [1]. A second-order connection on a $C^{\infty}$ manifold $M$ gives rise to a structure which resembles an immersion of $M$ into another Riemannian manifold. We call this an almost submanifold $(A S)$ structure, and call the structure an almost hypersurface $(A H)$ structure if an additional condition analogous to the codimension of $M$ being one is satisfied. As was noted in the previous paper, sometimes this $A H$ structure in fact satisfies the integrability conditions for an immession of $M$ into $R^{n+1}$. However, this realization as an immersion is not always possible in cases where the vector field analogous to a normal vector field vanishes at certain singular points. In the first part of this paper, we prove a more general integrability theorem, which considers isolated singular points, and we also study curvature properties of $A H$ structures at singular points. The second part of this paper introduces the concept of a realizable structure, which resembles a generalized immersion, and we obtain certain curvature conditions necessary for such a structure to exist on $M$. Other curvature conditions imply that an $A S$ structure cannot be an $A H$ structure, and two such theorems are included in this section. The last section of this paper states an equivalence principle between $A S$ structures and immersions of $M$ into another Riemannian manifold, and the principle is used to give immediate proofs of two theorems which simply translate theorems about hypersurfaces to theorems about $A H$ structures.

\section{Preliminary remarks and definitions}

In this section, we will outline the results of [1] used in this paper. A second-order connection on $M$, as defined originally in [2], determines a vector bundle structure on ${ }^{2} M$, such that ${ }^{2} M \simeq T M \oplus T M$. The first factor of $T M$ we call the horizontal bundle and identify with the tangent space of $M$; the second we call the vertical bundle. Henceforth, the letters $W, X, Y$, and $Z$ will always denote horizontal vector fields or horizontal vectors, $A$ and $B$ will denote sections of ${ }^{2} M$, and $\xi$ will be a vertical vector field or vertical vector. The second-order connection described above determines a covariant differ-

Communited by K. Yano, September 15, 1975. 
entiation $\tilde{\nabla}$ of a section $A$ of ${ }^{2} M$. Now let $\langle$,$\rangle be a fiber metric on { }_{0}^{2} \pi:{ }^{2} M$ $\rightarrow M$, such that the vertical and horizontal subbundles are orthogonal. We may then define an operator $\nabla^{\prime}$ in terms of $\tilde{V}$ by

$$
\begin{gathered}
\nabla_{X}^{\prime} Y=\tilde{\nabla}_{X} Y=\nabla_{X} Y+\alpha(X, Y), \\
\nabla_{X}^{\prime} \xi=-\mathscr{A}_{\xi}(X)+\tilde{\nabla}_{X} \xi=-\mathscr{A}_{\xi}(X)+D_{X} \xi .
\end{gathered}
$$

In the first equation of (1), the horizontal component $\nabla_{X} Y$ is the covariant derivative of the first-order connection induced by $\tilde{V}$, and the vertical component $\alpha(X, Y)$, which we call the second fundamental form, is bilinear. In the second equation of (1), $D$ is a connection in the vertical bundle, and the horizontal vector field $\mathscr{A}_{\xi}(X)$ is defined by

$$
\left\langle\mathscr{A}_{\xi}(X), Y\right\rangle=\langle\alpha(X, Y), \xi\rangle
$$

for all $Y$. The operator $\nabla^{\prime}$ is called an almost submanifold or $A S$ structure on ${ }^{2} M$. We shall also assume that $\nabla^{\prime}$ is Riemannian (see [1] for definitions); this implies that $\alpha(X, Y)=\alpha(Y, X)$.

We now define the first vertical space $V_{1}(x)$ of an $A S$ structure at $x \in M$ by

$$
V_{1}(x)=\operatorname{span}\left\{\alpha(X, Y) \mid X, Y \in M_{x}\right\}
$$

If $V_{1}(x)$ has maximum dimension $l$ at any point $X \in M$, we will call $l$ the pseudocodimension of $M$. If $l=1$, we call the $A S$ structure an almost hypersurface or $A H$ structure. In this case, we define a normal vector $\xi_{x}$ by orthonormalizing $\alpha(X, Y)$ where $\alpha \neq \equiv$, and by setting $\xi_{x}=0$ where $\alpha \equiv 0$. Moreover, we define $\mathscr{A}(X)=\mathscr{A}_{\xi}(X)$ for this $\xi$. It is proved in [1] that $D_{X} \xi$ and $\xi$ are orthogonal ; hence considering only tangent vectors and vectors in the first vertical space we redefine $\nabla^{\prime}$ by

$$
\begin{gathered}
\nabla_{X}^{\prime} Y=\nabla_{X} Y+\alpha(X, Y) \\
\nabla_{X}^{\prime} \xi=-\mathscr{A}(X)
\end{gathered}
$$

This $\mathscr{A}$ is very similar to a Weingarten map for an immersed hypersurface. We also define $h(X, Y)=\|\alpha(X, Y)\|$ and note that

$$
\alpha(X, Y)=h(X, Y) \xi \text {. }
$$

We define a singular point of an $A H$ structure as a point $x \in M$ such that $\alpha_{x}(X, Y) \equiv 0$ for all horizontal vectors $X$ and $Y$. At such a point, $\mathscr{A}$ may not be $C^{\infty}$, since (2) defining $\mathscr{A}$ can be rewritten as

$$
\langle\mathscr{A}(X), Y\rangle=\|\alpha(X, Y)\|=h(X, Y),
$$

and the length function is not $C^{\infty}$ at zero. However, since the length function 
is continuous at zero, $\mathscr{A}(X)$ will at least be contiunous for any differentiable vector field $X$.

The curvature tensor of an $A S$ structure $\nabla^{\prime}$ is defined as

$$
R^{\prime}(X, Y) A=\nabla_{X}^{\prime} \nabla_{Y}^{\prime} A-\nabla_{Y}^{\prime} \nabla_{X}^{\prime} A-\nabla_{[X, Y]}^{\prime} A
$$

By a standard calculation, (5) implies the equation analogous to the Gauss equation,

$$
\begin{aligned}
\left\langle R^{\prime}(X, Y) Z, W\right\rangle= & \langle R(X, Y) Z, W\rangle+\langle\alpha(X, Z), \alpha(Y, W)\rangle \\
& -\langle\alpha(Y, Z), \alpha(X, W)\rangle .
\end{aligned}
$$

In the case of an $A H$ structure, the curvature tensor $R^{\prime}$ is not well-defined on sections $A$ of ${ }^{2} M$, since the definition of $R^{\prime}$ given in (5) would involve taking derivatives of $\mathscr{A}(X)$, which is not differentiable at singular points. In fact, we cannot even define $R^{\prime}$ on vectors in $T(M)$ at singular points, since at a singular point we cannot write $\alpha(X, Y)$ as $f \cdot \xi$, where $f$ is a differentiable function. However, the right-hand side of (6) does make sence at singular points, so for an $A H$ structure with singular points, we define $\left\langle R^{\prime}(X, Y) Z, W\right\rangle$ by the right-hand side of (6). This makes $\left\langle R^{\prime}(X, Y) Z, W\right\rangle$ a $C^{\infty}$ operator on tangent vectors $X, Y, Z$, and $W$ for any $A H$ structure.

By an equation analogous to the Codazzi equation, the vertical component of $R^{\prime}(X, Y) Z$, at a nonsingular point of an $A H$ structure, is

$$
\left(\nabla_{X} h\right)(Y, Z) \xi-\left(\nabla_{Y} h\right)(X, Z) \xi \text {. }
$$

At a singular point, the expression in (7) is not defined. Finally, given two linearly independent horizontal vectors $X$ and $Y$, we define the sectional curvature $k^{\prime}(X, Y)$ of an $A S$ structure in the plane spanned by $X$ and $Y$ to be $\left\langle R^{\prime}(X, Y) Y, X\right\rangle$. By (6) we have

$$
k^{\prime}(X, Y)=k(X, Y)+\langle\alpha(X, Y), \alpha(X, Y)\rangle-\langle\alpha(X, X), \alpha(Y, Y)\rangle,
$$

where $k(X, Y)$ is the usual sectional curvature of $M$ in the plane spanned by $X$ and $Y$. Again, for an $A H$ structure with singular points we define $k^{\prime}(X, Y)$ by the right-hand side of (8). This makes $k^{\prime}(X, Y)$ a $C^{\infty}$ operator on tangent vectors $X$ and $Y$ for any $A H$ structure.

\section{Singular points of almost hypersurface structures}

Away from singular points, a theorem in [1] shows that an $A H$ structure on $M$ is integrable - that is, can be identified with an immersion of $M$ as a hypersurface of Euclidean space-if and only if $R^{\prime} \equiv 0$. However, as noted above, one of the integrability conditions, (7), breaks down at singular points. The following theorem summarizes the situation for a manifold with isolated singular points. 
Theorem 1. Suppose that $M$ is a simply connected and connected $C^{\infty} n$ dimensional manifold, which bears a Riemannian AH structure with $R^{\prime} \equiv 0$, and whose associated tensor $\mathscr{A}$ is $C^{\infty}$ except on a set $A$ of isolated singularities, which do not simply disconnect $M$. Then $M$ is isometrically imbeddable as a $C^{1}$ submanifold of $R^{n+1}$. Moreover, this submanifold admits a second fundamental form which agrees with $\mathscr{A}$.

Proof. The set $M-A$ is a connected and simply connected $C^{\infty}$ manifold such that $\mathscr{A}$ satisfies the Gauss-Codazzi equations. Hence by the fundamental theorem for hypersurfaces there is an isometric imbedding of $M-A$ into $R^{n+1}$ (which we also denote by $M-A$ ) such that $\mathscr{A}$ is the second fundamental form. We may fill in the holes in $M-A$ introduced in this procedure by taking limit points of $M-A$ in $R^{n+1}$ (this is possible since the imbedding is isometric and thus the holes must be single points) and denote the result by $M$ again.

If $x \in A, \gamma:\left[t_{0}, t_{1}\right] \rightarrow M$ is a piecewise $C^{\infty}$ curve through $x$ (that is, $C^{\infty}$ except at $x$ ), and $N$ is the normal vector field on $M-A$ (which exists locally at least), then set

$$
N^{\prime}(t)= \begin{cases}\mathscr{A}(T(t)), & t \neq t^{\prime}, \\ 0, & t=t^{\prime}\end{cases}
$$

where $T(t)$ is the tangent vector to $\gamma$ except at $\gamma\left(t^{\prime}\right)=x$. If we take $N\left(t_{0}\right)=$ $N_{r\left(t_{0}\right)}$, then the right-hand side of (9) is continuous since $\mathscr{A}(T(t)) \rightarrow 0$ as $t \rightarrow t^{\prime}$. Thus there is a unique solution $N(t)$ to (9) which must agree with the normal vector field except at $\gamma\left(t^{\prime}\right)=x$, so that we may define a normal vector at $x$ by setting $N_{x}=N\left(t^{\prime}\right)$. That $N\left(t^{\prime}\right)$ is independent of $\gamma$ may be seen as follows: Let $\delta:\left[s_{0}, s_{1}\right] \rightarrow M$ be a second broken $C^{\infty}$ curve parametrized such that $s^{\prime}=t^{\prime}$ and $\gamma\left(t^{\prime}\right)=\delta\left(s^{\prime}\right)=x$. If the broken $C^{\infty}$ curve $\varepsilon:\left[t_{0}, s_{1}\right] \rightarrow M$ is defined by

$$
\varepsilon(t)= \begin{cases}\gamma(t), & \text { if } t_{0} \leq t \leq t^{\prime}=s^{\prime}, \\ \delta(t), & \text { if } t^{\prime}=s^{\prime}<t \leq s_{1}\end{cases}
$$

by repeating the above argument, then $N\left(t^{\prime}\right)=N\left(s^{\prime}\right)$ is the same for all of these curves. The normal field thus obtained is clearly $C^{1}$.

Choose a coordinate plane of $R^{n+1}$ (employing an isometry of $R^{n+1}$ if necessary) which contains $N_{x}$, such that the slope of the line containing $N_{x}$ in the coordinate plane exists and is not zero. Consider the coordinate curve on $M$ obtained by intersecting this coordinate plane with $M$. The orthogonal projection of $N$ along the coordinate curve into the coordinate plane is continuous and thus determines a continuous field of normal lines to the coordinate curve in a neighborhood of $x$, whose slope exists and is not zero. If $f$ is the equation of the coordinate (planar) curve and $s(u)$ is the slope of the normal line through $(u, f(u))$, then 


$$
f^{\prime}(u)=-1 / s(u)
$$

and we see that the coordinate curve has a continuous derivative in a neighborhood of $x$. Since the coordinate curves through $x$ are $C^{1}$ and $x \in A$ is arbitrary, we see that $M$ is a $C^{1}$ submanifold. The second fundamental form may be globally defined by taking its value to be zero on $A$.

The next theorem leads to examples of manifolds with no integrable $A H$ structure :

Theorem 2. At any singular point of an AH structure on $M$, all sectional curvatures of the AH structure are the sectional curvatures of $M$ itself.

Proof. Since $\alpha \equiv 0$ at any singular point, the result follows immediately from (8).

Corollary. On any even-dimensional ovaloid $N$, there is no global integrable AH structure whose first-order connection agrees with the Riemannian connection of $N$.

Proof. Since the ovaloid $N$ is homeomorphic to an even-dimensional sphere, whose Euler characteristic is nonzero, every tangent vector field on such an ovaloid must have a zero. So the vector $\xi$ of the $A H$ structure must vanish at some singular point - that is, the set of singular points is nonempty. Moreover, for any $A H$ structure, the set of singular points is closed. By Theorem 2 , since all sectional curvatures of an ovaloid are positive, $R^{\prime}$ cannot be equal to zero at any singular point and hence, by the continuity of $R^{\prime}$, cannot be equal to zero in a whole neighborhood of a singular point. If $N$ consists entirely of singular points, the $A H$ structure is clearly not integrable. If $N$ has nonsigular points, by taking a point on the boundary of the singular set, we see that $R^{\prime}$ is not zero at some nonsingular point, so that the $A H$ structure is again not integrable.

\section{Realizable structures}

Given an immersion of $M$ into $R^{n+1}$ with second fundamental form $h(X, Y)$ and a nonvanishing $C^{\infty}$ unit vector field $\xi$ on $M$, there is an $A H$ structure on ${ }^{2} M$, obtained by taking $\alpha(X, Y)=h(X, Y) \xi$. Moreover, by Theorems 1 and 2, an $A H$ structure comes from such an immersion, if and only if $R^{\prime} \equiv 0$ and the $A H$ structure has no singular points. We may now ask which $A H$ structures arise from an immersion plus a vector field $\xi^{\prime}$ which is not of unit length, where again we define $\alpha(X, Y)=h(X, Y) \xi^{\prime}$. (In particular, $\xi^{\prime}$ might have a zerh.) This leads to the following definition.

Definition. An $A H$ structure on a Riemannian manifold $M$ will be said to be realizable if $\mathscr{A}=f \mathscr{A}^{\prime}$, where $\mathscr{A}^{\prime}$ is the Weingarten map coming from an isometric immersion of $M$ into $R^{n+1}$, and $f$ is a $C^{\infty}$ function on $M$. (Note that by defining $\alpha(X, Y)=h(X, Y) \xi^{\prime}, \xi^{\prime}$ not of unit length, we obtain such an $\mathscr{A}$.)

Theorem 3. Let $k^{\prime}(X, Y)$ be the sectional curvature of a realizable $A H$ 
structure in the plane spanned by $X$ and $Y$, and $k(X, Y)$ the sectional curvature of the same plane in the underlying manifold. Then

(i ) $k^{\prime}(X, Y) \leq k(X, Y)$ if $k(X, Y) \geq 0$, and $k^{\prime}(X, Y) \geq k(X, Y)$ if $k(X, Y)$ $\leq 0$, where the inequlity is strict unless either $k(X, Y)=0$ for the vectors $X$ and $Y$, or $f=0$ at the point,

(ii) at a point $x, k_{x}^{\prime}(X, Y)$ is a constant multiple of $k_{x}(X, Y)$, independent of the vectors $X$ and $Y$,

(iii) wherever $k(X, Y)$ is not equal to zero, the function $f$ is determined up to sign by the equation

$$
f^{2}=1-\frac{k^{\prime}(X, Y)}{k(X, Y)}
$$

Proof. By (2) and (8) together with the symmetry of $A^{\prime}$ and the Gauss equation for $A^{\prime}$ we have

$$
\begin{aligned}
\left\langle R^{\prime}(\right. & X, Y) Y, X\rangle \\
= & \langle R(X, Y) Y, X\rangle+\langle\mathscr{A}(X), Y\rangle\langle\mathscr{A}(Y), X\rangle-\langle\mathscr{A}(Y), Y\rangle\langle\mathscr{A}(X), X\rangle \\
= & \langle R(X, Y) Y, X\rangle \\
& +f^{2}\left(\left\langle\mathscr{A}^{\prime}(X), Y\right\rangle\left\langle\mathscr{A}^{\prime}(Y), X\right\rangle-\left\langle\mathscr{A}^{\prime}(Y), Y\right\rangle\left\langle\mathscr{A}^{\prime}(X), X\right\rangle\right) \\
= & \left(1-f^{2}\right)\langle R(X, Y) Y, X\rangle,
\end{aligned}
$$

which proves (i) and (ii); solving for $f^{2}$, we obtain (iii).

Partial converses to the above theorem, which show that the pseudocodimension is usually large if $k^{\prime} \geq k$, are the following.

Theorem 4. Suppose, at some point $p$ of $M$, there is a q-dimensional subspace $Q$ of $T_{p}(M)$ such that $k^{\prime}(X, Y) \geq k(X, Y)$ for all $X, Y$ in $Q$. If the real algebraic variety consisting of all $X$ in $Q$ such that $\alpha(X, X)=0$ has dimension $r$, the pseudocodimension of $M$ must be $\geq q-r$.

Proof. Restrict $\alpha$ to $Q$. Then $\alpha$ is a symmetric bilinear mapping of $R^{q} \times$ $R^{q} \rightarrow R^{s}$, where $R^{s}$ is the span of the image of $\alpha$. We must show $s \geq q-r$. By (8) we have

$$
k^{\prime}(X, Y)-k(X, Y)=-(\langle\alpha(X, X), \alpha(Y, Y)\rangle-\langle\alpha(X, Y), \alpha(X, Y)\rangle) .
$$

The following lemma completes the proof.

Lemma. Let $\alpha: R^{q} \times R^{q} \rightarrow R^{s}$ be a symmetric bilinear mapping. If

$$
\langle\alpha(X, X), \alpha(Y, Y)\rangle-\langle\alpha(X, Y), \alpha(X, Y)\rangle \leq 0
$$

for all $X, Y \in R^{q}$, and the real algebraic variety $V$ consisting of all $X$ in $R^{q}$ such that $\alpha(X, X)=0$ has dimension $r$, then $s \geq q-r$.

Proof. Extend $\alpha$ to a symmetric complex bilinear map of $C^{q} \times C^{q} \rightarrow C^{s}$. 
Consider the equation $\alpha(Z, Z)=0, Z \in C^{q}$. This is equivalent to a set of $s$ quadratic equations

$$
\alpha^{1}(Z, Z)=0, \cdots, \alpha^{s}(Z, Z)=0 .
$$

The set $S$ of solutions by the dimension theorem for complex algebraic varieties has (complex) dimension $d \geq p-s$. If $s \geq q$, we are done. If $s<q$, we choose any regular point $P \in S$. This point $P$ has a neighborhood $U$ which is a $d$-dimensional complex analytic submanifold of $C^{q}$. Thus there is a map $f$ : $C^{d} \rightarrow U \subset C^{q}$ such that some $d \times d$ subdeterminant of the $d \times q$ (complex) Jacobian is not zero in a neighborhood of $P$. So we may choose $d$ of the standard coordinates of $C^{q}$, which for convenience we relabel as $Z_{1}$ through $Z_{d}$, over which $U$ projects diffeomorphically onto an open subset $U^{\prime}$ of the complex subspace $V=\left\{Z_{1}, \cdots, Z_{d}, 0, \cdots, 0\right\}$. We let $R e: C^{q} \rightarrow R^{q}$ be the map which takes $\left\{Z_{1}, \cdots, Z_{q}\right\}$ into $\left\{X_{1}, \cdots, X_{q}\right\}$, where each $Z_{j}=X_{j}+i Y_{j}$. Then clearly $\operatorname{Re}(U)$ projects diffeomorphically down to $\operatorname{Re}\left(U^{\prime}\right)$, so $\operatorname{Re}(U)$ has (real) dimension $d$, as well. But $\operatorname{Re}(U)$ is a subset of $V$ by the following argument: Let $\tilde{Z}=\tilde{X}+i \tilde{Y}$ be any point in $U$. Then

$$
0=\alpha(\tilde{Z}, \tilde{Z})=\alpha(\tilde{X}, \tilde{X})-\alpha(\tilde{Y}, \tilde{Y})+2 i \alpha(\tilde{X}, \tilde{Y}) .
$$

So $\alpha(\tilde{X}, \tilde{X})=\alpha(\tilde{Y}, \tilde{Y})$ and $\alpha(\tilde{X}, \tilde{Y})=0$. By (14), this implies that $\alpha(\tilde{X}, \tilde{X})$ $=\alpha(\tilde{Y}, \tilde{Y})=0$; i.e., $\tilde{X}$ and $\tilde{Y}$ are both in $V$. So, $\operatorname{Re}(U)$ is a subset of $V$, and hence $r \geq d$. Since $d \geq q-s$, we have $s \geq q-r$. The second theorem in this area is similar to a theorem of Otsuki [4] in the case of immersions in Riemannian spaces.

Theorem 5. Let $M$ be a manifold admitting an AS structure. If, for some point $p \in M, k^{\prime}(X, Y)>k(X, Y)$ for all pairs of linearly independent vectors $X$ and $Y$ in a q-dimensional subspace $Q$ of $T_{p}(M)$, then the pseudocodimension of the $A S$ structure on $M$ is $\geq q-1$.

Proof. The hypothesis implies, as in the previous theorem, that

$$
\langle\alpha(X, X), \alpha(Y, Y)\rangle-\langle\alpha(X, Y), \alpha(X, Y\rangle<0 \text {. }
$$

for $X$ and $Y$ linearly independent. Assume that the dimension of the image of $\alpha$ is $<q-1$. The lemma stated in the last proof shows that there is a nonzero vector $X \in Q$ such that $\alpha(X, X)=0$. Consider the linear transformation given by $Z \rightarrow \alpha(X, Z)$. Since the dimension of the image of $\alpha$ is $<q$ -1 , and since dimension (null space) + dimension (image) $=$ dimension (domain), there is a vector $Y$, linearly independent from $X$, such that $\alpha(X, Y)$ $=0$. Substituting $X$ and $Y$ into (15) we obtain a contradiction.

Corollary. Let $M$ be a Riemannian manifold. If the sectional curvatures of a 3-dimensional subspace of $T_{p}(M)$ are all negative, for some point $p \in M$, then any realizable structure on $M$ whose first-order connection agrees with 
the Riemannian connection on $M$ must have a singular point at $p$. In particular, if the sectional curvatures of $M$ are all negative, and the dimension of $M$ is $\geq 3$, then the only realizable structure on $M$ is the trivial one, $\alpha(X, Y) \equiv 0$.

Proof. This follows immediately from part (i) of Theorem 3.

Remark. An example of a manifold with an $A S$ structure whose pseudocodimension is $>1$ is given by taking a manifold $M$ admitting two metrics $g$ and $g^{\prime}$. Let $\alpha$ be the difference tensor of the two metrics, and let the connections in the vertical and horizontal bundles be (say) the Riemannian connections coming from $g$ and $g^{\prime}$ respectively. To obtain the example, let $M^{n}$ be an ovaloid, and $g$ the first fundamental form of $M$. Since the second fundamental form of an ovaloid is positive definite, we may let $g^{\prime}$ be the second fundamental form of $M$. We recall that the rank of the difference tensor is defined as follows : Let $\left\{e_{\alpha}\right\}$ be a local orthonormal frame for $g$, and let the components of the difference tensor $\alpha_{b c}^{a}$ be defined by the equation

$$
\alpha\left(e_{b}, e_{c}\right)=\sum_{a} \alpha_{b c}^{a} e_{a}
$$

Define a bilinear form $B$ by setting

$$
B\left(e_{s}, e_{t}\right)=\sum_{u, v} \alpha_{u v}^{s} \alpha_{u v}^{t} .
$$

We define the rank of $\alpha$ to be the rank of $B$ as a bilinear form. (See [5] for a more intrinsic definition of $B$.)

Lemma. The rank of $\alpha$ at $x \leq$ the dimension of $V_{1}(x)$.

Proof. Let $q$ be the dimension of $V_{1}(x)$, and let the frame $\left\{e_{i}\right\}$ have the property that $e_{q+1}, \cdots, e_{m}$ span the complement to $V_{1}(x)$. By (16), $\alpha_{b c}^{a}=0$ for $a>q$. Hence, by (17), $B\left(e_{b}, e_{c}\right)=0$ for $b$ or $c>q$, so the rank of $B$ is $\leq q$.

Using this lemma, we may restate a theorem in [5] as follows:

Theorem. For an ovaloid $N^{m}$, if the dimension of $V(x) \leq q$ on $x$ in an open subset $U$ of $N$, then $U$ is at least $(m-q)$-umbilical, and the principal curvature associated to the $(m-q)$-umbilical directions is constant $(q \neq m$ or $m-1)$.

Now take an ovaloid $N^{n}$ on which the principal curvatures are not equal on any open set, such as an ellipsoid

$$
\sum_{i=1}^{n+1} X_{i}^{2} / a_{i}^{2}=1,
$$

where $a_{i}^{\prime}$ s are all unequal. Then, for almost all points $x$ in $N^{n}$, the dimension of $V_{1}(x)>n-2$, since otherwise $N^{n}$ would be at least 2-umbilic on an open set. So the pseudocodimension of $N^{n}$ is at least $n-1$. 


\section{5. $A H$ structures as immersions}

In many cases, we may simply regard $R^{\prime}$ as the curvature of an imaginary immersing space for $M$ and note that proofs for immersed submanifolds go through almost verbatim to give proofs of similar theorems about $A H$ or $A S$ structures. As examples of this technique we quote the following two theorems.

Theorem 6. Let $M$ be a manifold admitting an AH structure, and $\mathscr{A}$ the associated tensor of this structure. Let $t(x)$ be the type number of $\mathscr{A}$ at $x-$ that is, the rank of the linear transformation $\mathscr{A}$ at $x$. Let $R^{\prime}(X, Y)$ and $R(X, Y)$ be the curvature transformations arising from the curvature tensors $R^{\prime}$ and $R$, respectively. Then

(i) $t(x)=0$ or $1 \Leftrightarrow R^{\prime}(X, Y)=R(X, Y)$ for all plane sections at $x$,

(ii) $t(x) \geq 2 \Leftrightarrow t(x)=n-\operatorname{dim} T_{x}^{*}$, where

$$
T_{x}^{*}=\left\{X \in T_{x}(M): R(X, Y)=R^{\prime}(X, Y), \forall Y \in T_{x}(M)\right\} .
$$

Theorem 7. Let $f_{1}$ and $f_{2}$ be AH structures on ${ }^{2} M$ inducing the same metric on $M$, and let $R_{f_{1}}^{\prime}(X, Y)=R_{f_{2}}^{\prime}(X, Y)$ for all plane sections of $M$. If the type number of $\mathscr{A}_{f_{1}} \geq 3$, then $\mathscr{A}_{f_{1}}= \pm \mathscr{A}_{f_{2}}$.

The proofs of Theorems 6 and 7 can be easily deduced from the fact that the relative curvature $R(X, Y)-R^{\prime}(X, Y)$ plays the same role as $R(X, Y)$ does for a hypersurface immersed in Euclidean space, where the counterpart of the curvature $R^{\prime}$ of the immersing space is equal to zero. We then note that the proofs of the analogous theorems in Euclidean space [3, Theorems (6.1) and (6.2), pp. 42 and 43] make no use of the special properties (such as homogeneity) of Euclidean space. Moreover, the proofs rely only on the Gauss equation, which is formally identical to (6). So those proofs go through to this case. Other theorems can be proved similarly.

This equivalence principle can also be reversed; certain theorems about $A S$ structures can yield theorems about submanifolds of Euclidean space. As an example, the reader may easily modify the proof of Theorem 4 to obtain the following extension of [3, Theorem 4.7, p. 28].

Theorem 8. Let $M$ be an n-dimensional Riemannian manifold isometrically immersed in $R^{n+p}$, and let $\alpha(X, Y)$ be the second fundamental form of $M$. Then $p \geq m-d$, if there is a point $x$ of $M$ such that

(i) the tangent space $T_{x}(M)$ contains an m-dimensional subspace $T_{x}^{\prime}$ such that the sectional curvature for any plane in $T_{x}^{\prime}$ is nonpositive, and

(ii) the set of vectors $X \in T_{x}^{\prime}$ such that $\alpha(X, X)=0$ forms a real algebraic variety of dimension $d$. 


\section{References}

[ 1 ] R. H. Bowman, Almost submanifold structures, J. Differential Geometry 9 (1974) 537-546.

[ 2 ] - Second order connections, J. Differential Geometry 7 (1972) 549-561.

[ 3 ] S. Kobayashi \& K. Nomizu, Foundations of differential geometry, Vol. II, Interscience, New York, 1969.

[4] T. Otsuki, Isometric imbedding of Riemannian manifolds in a Riemannian manifold, J. Math. Soc. Japan 6 (1954) 221-234.

[5] R. B. Gardner, Subscalar pairs of metrics and hypersurfaces with a nondegenerate second fundamental form, J. Differential Geometry 6 (1972) 437-458.

Arkansas State University Arkansas College 